Cell Research (2000),10, 59-69

\title{
$\gamma$-Aminobutyric acid transporter (GAT1) overexpression in mouse affects the testicular morphology
}

\author{
Ma Ying Hua ${ }^{1}$, Jia Hua Hu${ }^{1}$, Xiao Gang Zhou ${ }^{1}$, Zhen Tong \\ $\mathrm{MEI}^{2}$, JiAn FEI ${ }^{1, *}$, Li He GuO ${ }^{1, *}$ \\ ${ }^{1}$ Shanghai Institute of Cell Biology, Chinese Academy of Science, \\ Shanghai 200031, China \\ ${ }^{2}$ Shanghai Institute of Physiology, Chinese Academy of Science, \\ Shanghai 200031, China
}

\begin{abstract}
$\gamma$-Aminobutyric acid and GABAergic receptors were previously reported to be distributed in reproductive systems besides CNS and predicted to participate in the modulation of testicular function. $\gamma$-Aminobutyric acid transporter was implicated to be involved in this process. However, the potential role of $\gamma$-aminobutyric transporter in testis has not been explored. In this study, we investigated the existence of mouse gaminobutyric acid transporter subtype I (mGAT1) in testis. Wild-type and transgenic mice, which overexpressing mGAT1 in a variety of tissues, especially in testis, were primarily studied to approach the profile of mGAT1 in testis. Mice with overexpressed mGAT1 develop normally but with reduced mass and size of testis as compared with wild-type. Testicular morphology of transgenic mice exhibited overt abnormalities including focal damage of the spermatogenic epithelium accompanied by capillaries proliferation and increased diameter of seminiferous tubules lumen. Reduced number of spermatids was also found in some seminiferous tubules. Our results clearly demonstrate the presence of GAT1 in mouse testis and imply that GAT1 is possibly involved in testicular function.
\end{abstract}

Key words: $\gamma$-aminobutyric acid transporter, testicular morphology, mouse.

* Corresponding auythor. E-mail: mhzhang@sunm.shcnc.ac.cn 
GAT1 overexpression alters testicular morphology in mouse

\section{INTRODUCTION}

As the predominant inhibitory neurotransmitter in the vertebrate, $\gamma$-aminobutyric acid (GABA) in central nervous system (CNS) was intensively studied. GABA actions are mediated by the ionotropic $\mathrm{GABA}_{A} / \mathrm{GABA}_{C}$ receptors, as well as the metabotropic $\mathrm{GABA}_{B}$ receptors. Recently, GABA was found in gonads and accessory reproductive organs, and a direct effect on steroidogenesis and sperm viability and motility has been described[1]. The existence of peripheral-type benzodiazepine receptors $(\mathrm{PBR})$ in testicular interstitial cells was characterized[2]. Glutamic acid and glutamate decarboxylase (GAD) which produce GABA from glutamic acid, the main source of GABA via deamination, were also detected in testis[1]. All these studies mentioned above hinted at the physiological role of GABA network in reproductive system.

On the other side, the termination of GABA synaptic transmission is catalyzed by transporters which are ubiquitously distributed in various parts of brain but has not yet been detected in reproductive system so far. Aanesen A. et al[3] indicated that a highaffinity $\gamma$-aminobutyric acid transporter protein $\mathrm{m}$ ay be possibly involved in GABA uptake in human spermatozoa, but without direct evidence. In the present study, we demonstrated, for the first time, that $\gamma$-aminobutyric acid transporter subtype 1 (mGAT1), which can rapidly and specifically take up GABA into pre-synaptic terminal or surrounding glial cells in CNS, was also present in mouse testis. To approach the functional role of GAT1 in testis, a model of transgenic mice over-expressing mGAT1 which have been identified previously (unpublished Data) was used. Here, we reported the influence of GAT1 on testicular morphology

\section{MATERIALS AND METHODS}

\section{Animals}

C57BL mice carrying mGAT1 cDNA have been constructed and identified (unpublished Data). Mouse GAT1 cDNA containing whole mRNA coding region, which was screened from the mouse brain cDNA/ $\lambda$ phage library, was cloned into pCDNA3 (EcoRI-ApaI) under the control of human cytomegalovirus (HCMV) promoter/enhancer. DNA constructs were microinjected into the fertilized eggs of C57BL mice. Polymerase chain reaction (PCR) amplification and Southern blot hybridization of tail DNA samples verified the integration of variable copy numbers of transgene into the genomes of founder mice and their progeny. Reverse transcription-polymerase chain reaction (RT-PCR) analysis and Nothern blot hybridization of mRNA samples from various tissues characterized the expression pattern of transgene. Age- and sex- matched wild-type C57BL mice were served as controls. Mice were housed in groups $(<4$ per cage $)$ in temperature and humidity-controlled environment with a 12-h light/12-h dark rhythm. All mice had free access to food and water.

\section{Reverse transcription-Polymerase Chain Reaction (RT-CR) analysis}

Testes were removed from wild-type and homozygous transgenic mice after cervical dislocation. Whole RNA was extracted with Trizol reagent (GIBCO BRL) as detailed by the manufacturer. RNA integrity was identified by formaldehyde-electrophoresis. RNA sample was thoroughly treated by incubating with RNA-Free DNase $(5 \mathrm{u} /$ per $\mu \mathrm{g})$ for $45 \mathrm{~min}$ at $37^{\circ} \mathrm{C}$ before reverse- 
transcription performed with GIBCO Kit. mGAT1 was amplified by PCR with $64^{\circ} \mathrm{C}$ as annealing temperature. PCR primers (5' -end: 5' -ACCAAGCTTAGGCTGCAAAGCTGCTG-3'; 3 ' end: 5' -ACGCCTTTGAACATGGGCGCCAG- 3') were designed to match nucleotides from (92) to (+375) of mouse GAT1 cDNA[4]. GAPDH mRNA was co-detected by PCR with primers (5'end: 5' -ACGACCCCTTCATTGACC-3'; 3' -end: 5' -AGACACACACAGTAGACTCCACG3') which spanned $210 \mathrm{bp}$ nucleotides within the coding sequence for GAPDH. The amount of RT-PCR product from GAPDH mRNA was refered to semiquantify the expression level of mGAT1. RT procedure omitted RNA samples were directly amplified by PCR with 5 -fold amounts of the same aliquot to demonstrate the amplified product was cDNA-based instead of genomic DNAbased product. mGAT1 RT-PCR products from both wild-type and transgenic mice were purified and subsequently sequenced by using a primer specific to 5 ' -terminal sequence of mGAT1 cDNA.

\section{Immunofluorescence analysis}

Seven-month-old mice were anesthetized and perfused with chilled 95\% ethanol. After perfusion, testes were removed and subsequently rinsed with $30 \%$ sucrose in phosphate-buffered saline (PBS) and frozen in OCT. $20 \mu \mathrm{m}$-thick frozen sections were cut and then rinsed in $0.01 \mathrm{M}$ PBS. In staining procedure, cryostat sections were firstly blocked with $10 \%$ goat serum plus $0.1 \%$ Triton X-100 in PBS and incubated with rabbit anti-mGAT1 antibody[5] at $4^{\circ} \mathrm{C}$ overnight. After incubation, the slides were rinsed in PBS for $30 \mathrm{~min}$ and exposed to biotinlated goat anti-rabbit IgG (Santa-Cruz) for $45 \mathrm{~min}$ at room temperature and then rinsed in PBS. Slides subsequently incubated with Fluorescent Extravidin (Santa-Cruz) for $45 \mathrm{~min}$ at room temperature. Slides were examined and photographed under Olympus fluorescence microscope.

\section{Histology analysis}

Seven-month-old mice were anesthetized and perfused with $4 \%$ paraformaldehyde in PBS. After perfusion, testes were removed and then dehydrated through graded ethanol and cleared with xylene, subsequently embedded in paraffin. $5 \mu \mathrm{m}$-thick sections were cut on Leitz microtome. Slides were stained with haematoxylin and eosin, then examined and photographed under Leitz microscope.

The diameters of the seminiferous tubules and their lumens were measured by fitting a graticule of a calibrated Linear Scale in the $\times 10$ eyepiece of Leitz microscope at objective lens $\times$ 40. Only circular and near circular tubules were assessed. The height of the seminiferous epithelium was calculated by subtracting the lumen diameter from the tubule diameter. All tissue variables were assessed by viewing four randomly chosen areas per section. Four sections per mouse from both wild-type $(n=7)$ and transgenic mice $(n=6)$ were measured. The mean data was analyzed statistically. Testicular morphology of both wild-type and transgenic mice was represented by one section.

\section{Testicular weight}

Seven-month-old mice were sacrificed and dissected to remove testis. Testicular weight was assessed by electron-balance.

\section{Determination of ${ }^{3} \mathrm{H}-\mathrm{GABA}$ uptake in testis}

Testicular cell suspension prepared by filtration with nylon membrane was pre-incubated in aCSF buffer (containing in $\mathrm{mM}: \mathrm{NaCl} 126.6 ; \mathrm{NaCO}_{3} 27.4 ; \mathrm{KCl} 2.4 ; \mathrm{KH}_{2} \mathrm{PO}_{4} 0.49 ; \mathrm{CaCl}_{2} 1.2$; $\mathrm{MgCl}_{2} \cdot 6 \mathrm{H}_{2} \mathrm{O} 0.83 ; \mathrm{Na}_{2} \mathrm{HPO}_{4} 0.49$; D-glucose 7.1 ; gassed with $95 \% \mathrm{O}_{2} / 5 \% \mathrm{CO}_{2} ; \mathrm{pH} 7.2-7.4$ ) for $5 \mathrm{~min}$ at $37^{\circ} \mathrm{C}$. After that, uptake was initiated by the addition of a mixture of cold and tritiated GABA $\left({ }^{3} \mathrm{H}-\mathrm{GABA}\right.$ specific activity: $\left.98 \mathrm{ci} / \mathrm{mM}\right)$ and incubated further for $10 \mathrm{~min}$. Low-[Na $\mathrm{Na}^{+}$and high-[Na $\left.{ }^{+}\right]$ analysis of uptake in wild-type was carried out separately to demonstrate that the GABA uptake capacity is sodium-dependent which is a characteristic possessed by GABA transporter. In this experiment, the different final concentration of GABA used was $0.04 \mu \mathrm{M}$ and $40 \mu \mathrm{M}$ respectively in a medium containing $4.2 \mathrm{nM}$ tritiated GABA. Modified aCSF buffer was served as Low-[ $\left.\mathrm{Na}^{+}\right]$ 
GAT1 overexpression alters testicular morphology in mouse

buffer, in which $\mathrm{NaCl}$ was substituted by $\mathrm{LiCl}$ with the same concentration. For kinetic analysis of uptake in both wild-type and transgenic mice, the amount of ${ }^{3} \mathrm{H}-\mathrm{GABA}$ used was kept constant and the different GABA used concentrations $(0.04-40 \mu \mathrm{M})$ were obtained by adding different amount of unlabelled GABA.

After 10 min incubation, uptake was terminated by vacuum filtration through filters. The GABA content of the filters was assayed by liquid scintillation counting, taking dilution factors into account. The same aliquot of testicular cell suspension was lysised with $2 \mathrm{~mol} / \mathrm{L} \mathrm{NaOH}$ to quantify the protein concentration. Uptake was expressed as $\mathrm{fmol} / \mathrm{mg}$ protein.

For kinetic data analysis, linear-fitting was done to calculate $\mathrm{Km}$ and Vmax values according to Michaelis-Menten equation $\left(\mathrm{V}=\mathrm{V}_{\max }[\mathrm{S}] /(\mathrm{Km}+[\mathrm{S}])\right)$.

\section{Statistical analysis}

Mean value and significant difference were analyzed by analysis of variance and student's t-test.

\section{RESULTS}

\section{GAT1 mRNA distribution in testis of wild-type and transgenic mice}

The distribution of GAT1 mRNA in testis of seven-month-old wild-type mice was revealed by RT-PCR amplification and sequence analysis. With specific primers matching to mGAT1, a 468 base-pair fragment was amplified (Fig 1, Lane 1). Simultaneously, we obtained a corresponded size product from brain mRNA via PCR with the same primers (Data not shown). Further sequence analysis indicates that the product from both brain and testis possessed identical sequence except that two nucleotides $[G(+105$ in brain

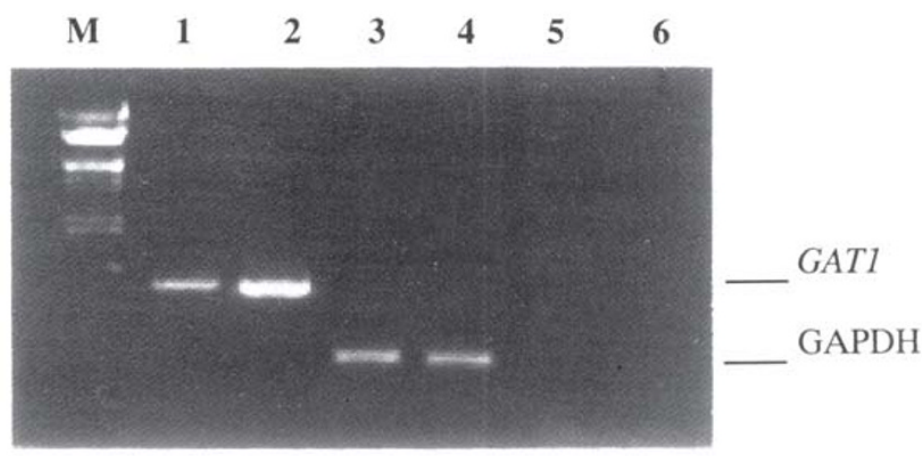

Fig 1.

Identification of GAT1 mRNA level in testis of both wild-type (Lane 1, 3, 5) and transgenic (Lane 2, 4, 6) mice. Specific primers to mGAT1 were used by semiquantitative RT-PCR analysis. Lane 1 shows the endogenous GAT1 expression in wild-type mice. Lane 2 shows the endogenous GAT1 and transgene expression in transgenic mice. Primers for GAPDH mRNA served to monitor quality and quantity of RNA in each sample (Lane 3, 4). Note that the samples loaded in lanes 1, 3 and 2, 4 were the same sample and with equal amount. RNA samples omitted reverse-transcription were directly amplified with GAT1specific primers to verify the absence of genomic DNA Contamination (Lane 5, 6). M $\lambda$ / EcoRI \& Hind III. 
$\mathrm{Ma}$ YH et al.
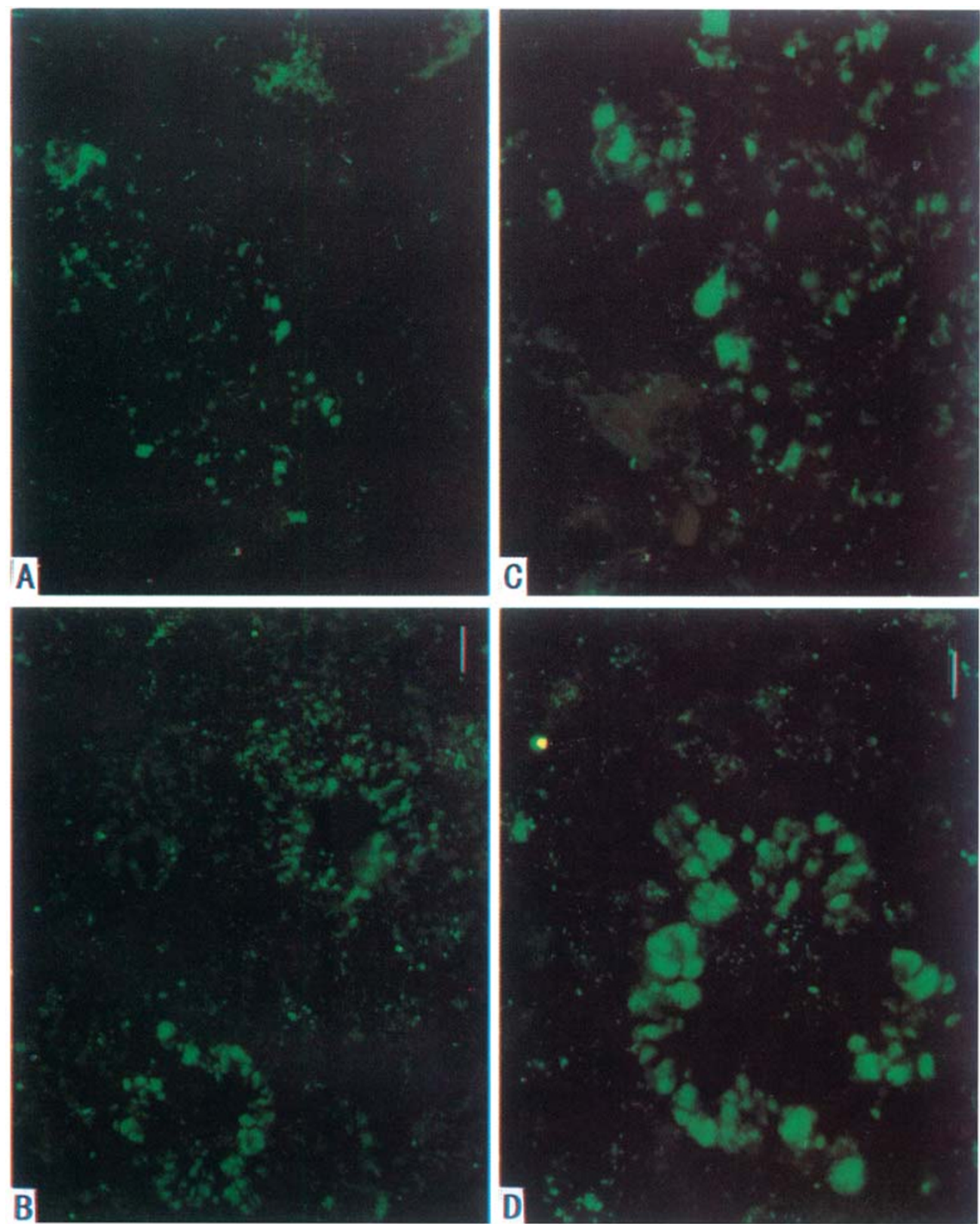

Fig 2.

Immunofluorescent detection showing GAT1 expressed in elongated spermatids and spermatozoa within the seminiferous tubules in both wild-type (A, C) and transgenic (B, D) mice. Obviously stronger signal in transgenic mice indicated the higher expression level of GAT1 compared with that in wild-type. Note the lumen of seminiferous tubules was full of relatively weaker GAT1positive spermatozoa in wild-type but much fewer in transgenic mice. E elongated spermatid So spermatozoa. Scale bars represent $20 \mu \mathrm{m}$ (A, B) and $10 \mu \mathrm{m}(\mathrm{C}, \mathrm{D})$ respectively. $20 \mu \mathrm{m}$-thickcryosections 
GAT1 overexpression alters testicular morphology in mouse

GAT1 mRNA)->A (+105 in testis GAT1 mRNA)] with synonymous amino acid mutation (Gln $>$ Gln) which might be introduced by low-fidelity Taq polymerase (Data not shown). With results mentioned above, we concluded that GAT1 was authentically expressed in mouse testis.

Transgene expression level in testis was assessed by semiquantative analysis. In RNA extraction from testis of transgenic mice, the GAT1 mRNA level was apparently higher (Fig 1, Lane 2) than that in wild-type mice (Fig 1, Lane 1), because the amount of PCR product reflected both the endogenous and transgenic GAT1 expression levels in transgenic mice. GAPDH mRNA levels, which were served as endogenous reference (Fig 1, Lane 3, 4) to monitor quantity and integrity of RNA in both samples, remained approximately identical. No amplification products formed from reverse-transcrption process omitted samples (Fig 1, Lane 5,6) verified that the specific product was derived from mRNA other than genomic DNA. Above results indicate that GAT1 was over-expressed in the testis of transgenic mice as compared with wild-type mice.

\section{GAT1 protein localization in testis of wild-type andtransgenic mice}

To locate GAT1 in specific testicular cell types, in situ immunofluorescence experiments were performed (Fig 2 ). High-affinity anti-mGAT1 antibody specifically against the C-terminal region of GAT1[5] was developed to probe the GAT1 in seminiferous tubules. Moderate immunoreactivity was found in elongated spermatids and spermatozoa within seminiferous epithelium. Spermatozoa within the lumen of tubule was also weakly labeled in wild-type mice (Fig 2A, C). Thus, expression of GAT1 was apparently confined to spermatids and spermatozoa.

Evaluation of immunofluorescence intensity by photoimager analysis indicated that the testis sections of transgenic mice (Fig 2B, D) possessed more intensive immunoreactivity than that of wild-type mice. To our surprise, the GAT1-positive signal was also limited to the spermatids and spermatozoa, which was identical to the expression pattern of endogenous GAT1. It might reflect that the expression pattern of transgene directed by human cytomegalovirus (HCMV) promoter possess the cell-type specific characteristic. In addition, no spermatozoa within the lumen of seminiferous tubules was labeled in wild-type testis.

Transgenic mice over-expressing GAT1 leads to abnormalities of testicular morphology

Abnormalities of testicular morphology in transgenic mice over-expressing GAT1 were revealed by histological observation. The testis of transgenic mice indicated many focal damages, in which some seminiferous tubules contained mostly spermatogenic cells while others were very vacuolated with reduced spermatogenic epithelium (Fig 3D, E, F).

Although the diameter of seminiferous tubule showed no difference $(\mathrm{P}>0.05)$ between transgenic mice and wild-type, the height of seminiferous epithelium in testis of transgenic mice reduced significantly $(\mathrm{P}<0.05)$, accompanied by conspicuously expanded 
$\mathrm{Ma}$ YH et al.

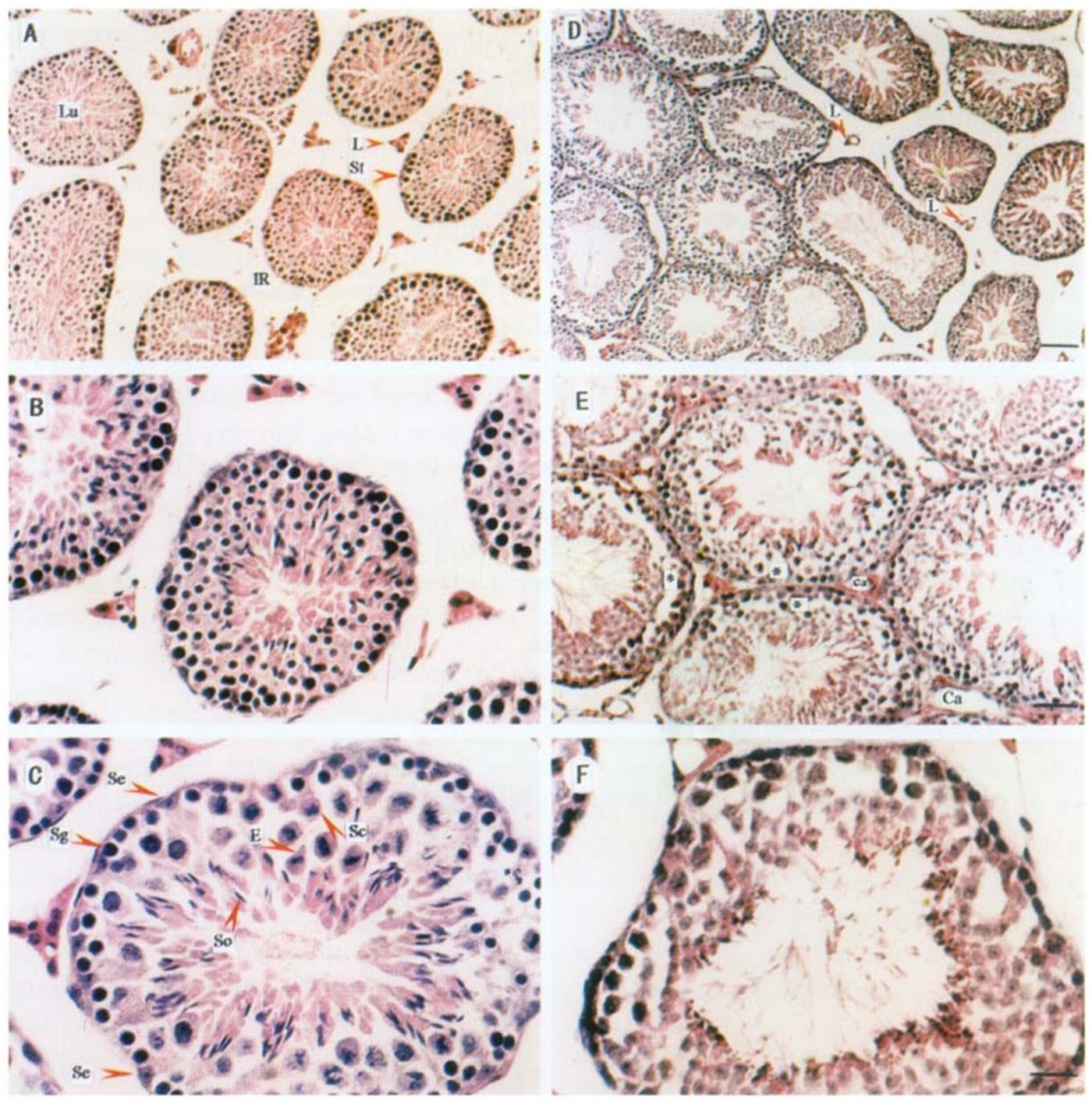

Fig 3.

Photomicrographs of the histomorphology of seminiferous tubules from both wild-type mice (A, $\mathrm{B}, \mathrm{C})$ and transgenic mice (D, E, F). Note the normal height of the spermatogenic epithelium and the diameter of lumen. Various stages of spermatogenic cells including spermatozoa in wild-type are indicated. In GAT1 overexpression mice, most seminiferous tubules showed markedly expanded lumen with reduced spermatogenic epithelium and less spermatozoa. The formation of striking vacuolization of tubules were occurred in some seminiferous tubles in transgenic mice. The interstitial region contains increased number of capillaries. Leydig cells were presented but atypically packed with lipid droplets. Sertoli cells appeared normal. St seminiferous tubules IR interstitial region $\mathbf{L}$ leydig cell $\mathbf{L u}$ lumen $\mathbf{S g}$ spermatogonia $\mathbf{S e}$ spermatocytes $\mathbf{E}$ elongated spermatid So spermatozoa Se sertoli cell Ca Capillaries * vacuolated cytoplasm. Scale bars represent $50 \mu \mathrm{m}$ (A, D) and $30 \mu \mathrm{m}(\mathrm{B}, \mathrm{E})$ and $15 \mu \mathrm{m}(\mathrm{C}, \mathrm{F})$ respectively. Paraffin embedded $5 \mu \mathrm{m}$-thick-sections 
GAT1 overexpression alters testicular morphology in mouse

lumen $(\mathrm{P}<0.01)$ (Tab 1). Compared with wild-type which generally showed a full complement of spermatogenic cells including spermatozoa within the seminiferous tubules, the reduction of seminiferous epithelium in transgenic mice indicated a depletion of spermatogenic cells. Furthermore, transgenic mice had fewer spermatids and spermatozoa per seminiferous tubule cross section than did the wild-type mice (Data not shown) (Fig $3 \mathrm{~F}, \mathrm{C})$.

In the testis of mice over-expressing GAT1, the interstitial region was featured with abnormally proliferated capillaries. Leydig cells were present within the interstitium, yet in marked contrast to the wild-type mice, some Leydig cells occasionally contained a lot

Tab 1. Morphological characteristics of testis in wild-type $(n=7)$ and transgenic mice $(\mathrm{n}=6)$.

\begin{tabular}{|c|c|c|}
\hline Variable & $\mathrm{Wt}$ & $\mathrm{Tg}$ \\
\hline Seminiferous tubule diameter $(\mu \mathrm{m})$ & $183.3 \pm 15.6$ & $195.0 \pm 20.8$ \\
\hline Seminiferous epithelium height $(\mu \mathrm{m})$ & $61.1 \pm 8.4$ & $50.7 \pm 12.4^{*}$ \\
\hline Lumen diameter & $58.5 \pm 14.9$ & $93.6 \pm 13.7^{* *}$ \\
\hline
\end{tabular}

Values are given as means \pm SEM, those values which are significantly different between wild-type (Wt) and transgenic ( $\mathrm{Tg}$ ) mice were indicated by $* \mathrm{P}<0.05$ and ${ }^{* *} \mathrm{P}<0.01$.
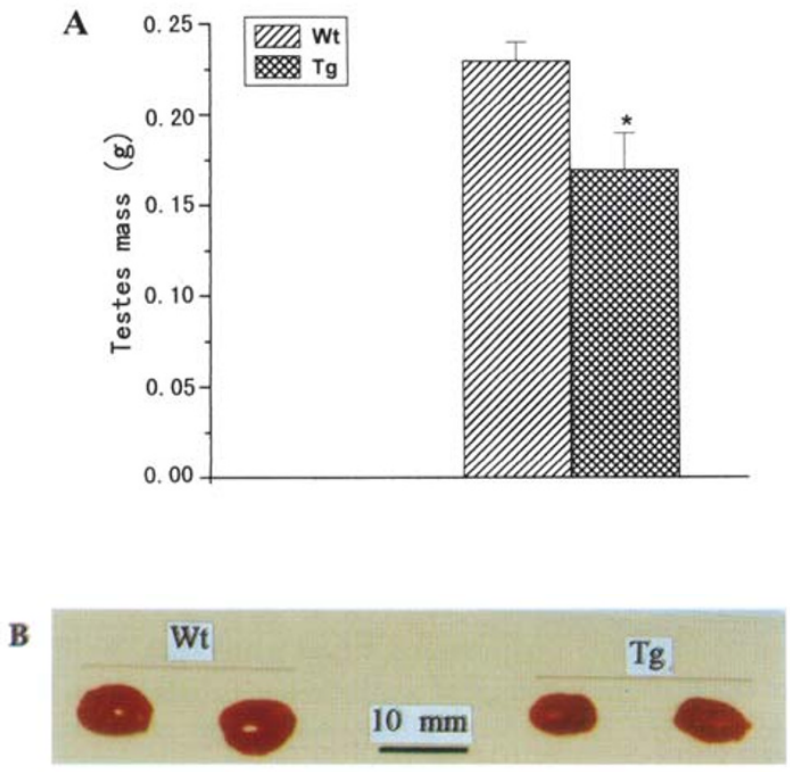

Fig 4.

Effect of GAT1 overexpression on testicular mass and size. A. Showing the testicular mass difference between wild-type and transgenic mice. Results are expressed as means \pm SEM (Wt $n=9$; Tg $n=8$ ). * indicates significantly different from wild-type $(P<0.01)$. B. Samples of left and right testes taken randomly from wild-type and transgenic mice. Scale bar is indicated. Wt wild-type Tg transgenic mice 
of lipid droplets in the cytoplasm. No morphological change was observed in sertoli cells of transgenic mice.

The effect of GAT1 over-expression on the mass and size of testis was also assessed statistically. The mean testicular mass of transgenic mice was reduced significantly ( $P$ $<0.01$ ) compared with that of wild-type (Fig 4A). Testicular size was also apparently reduced in short axis but not in long axis ( Fig 4B). Especially, testis of transgenic mice featured the loss of rigidity and elasticity which could be grossly evaluated by press with fingers, a difference which might be resulted from the alteration of testicular morphology.

\section{DISCUSSION}

$\gamma$-Aminobutyric acid has been previously found to be widely distributed in periphery besides nervous system and was suggested to possess comprehensive physiological role in these organs. Recently, a number of neurotransmitters are reported to be involved in the modulation of testicular function, including a stimulatory action of $\gamma$-amino-butyric acid on steroidogenesis in the rat testis[1]. Coexistence of GABAA and GABAB receptors in testicular interstitial cells was also indicated[2]. It is known that in CNS, neurotransmitter actions are mediated by specific receptors and terminated by high-affinity transporters, constituting together a complicated network.

Based on above mentioned findings, it is logical to infer that $\gamma$-aminobutyric acid transporter will be present in testis. Our results clearly demonstrated the constitutive expression of $\gamma$-aminobutyric acid transporter subtype 1 (GAT1) in mouse testis, which confined to spermatids and spermatozoa. We also primarily assessed the influence of increased amount of GAT1 on the testicular morphology with a transgenic model. The most striking feature of testis caused by GAT1 over-expression is the focal damage of the spermatogenic epithelium accompanied by capillaries proliferation which may be implicated in the onset of testicular pathologies that cause male infertility[6].

The reproductive performance of male of homozygous transgenic mice mated with a wild-type female was grossly observed. Transgenic mice with age under 2 mon showed no difference to age-matched wild-type in the number of pregnancies. But in mice older than 2-3 mon, the numbers of pregnancies were apparently lower when compared with the results from wild-type mice. This progressive reduction in pregnancy efficiency indicated that appropriate expression of GAT1 may be required for the maintenance of normal testicular function.

To investigate whether $\gamma$-amino-butyric acid transporters are involved in the GABA uptake process in testis, we performed ${ }^{3} \mathrm{H}$-GABA uptake experiments in the presence of low and high level of sodium concentration. As shown in Fig 5 , it is clearly demonstrated that GABA uptake capacity is sodium-dependent, which is one of the essential characteristic possessed by transporter. Km value determined by kinetic analysis (Data not shown) is also consistent with reported range $(1 \mathrm{mM}-4 \mathrm{mM})$ [7]. However, preliminary experi- 
GAT1 overexpression alters testicular morphology in mouse

ment indicated that testicular GABA uptake showed no significant difference between wildtype and transgenic mice which over-expressing GAT1 in testis (Data not shown). One of opinion suggested that the sodium-dependent GABA uptake in mouse testis may be also attributed to other GABA transporter subtypes other than GAT1. Other possibility is that the failure of increased uptake capacity in testis with over-expressed $\mathrm{mGAT1}$ may be

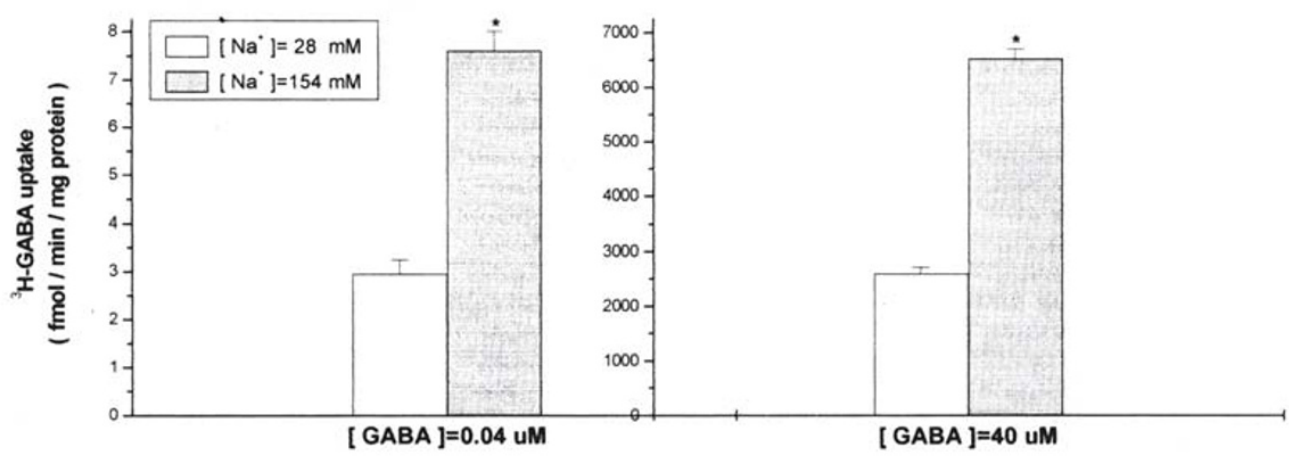

Fig 5.

The effect of sodium concentration on ${ }^{3} \mathrm{H}-\mathrm{GABA}$ uptake in testis was measured in the presence of $0.04 \mathrm{~mm}$ and $40 \mathrm{~mm}$ GABA respectively. Values are given as mean $\pm \operatorname{SEM}(\mathrm{n}=4) .{ }^{*}$ means significantly different from uptake at low-sodium concentration (student's t-test).

resulted from the morphology alteration as a compensatory effect. This may also imply that GAT1 has possibly other functions in testis.

In conclusion, the present study demonstrates the existence of GAT1 in mouse, it's over-expression results in alteration on testicular morphology.

\section{ACKNOWLEDGMENTS}

The authors are very grateful to Dr. Cai Guoqiang for his generous gift of anti-mGAT1 antibody. We thank prof. Wang Da for invaluable assistance with histology techniques. We also thank prof. Yan Yuanchang for his beneficial scientific interpretation and Miss Gao Lu for her technical assistance on this manuscript. This work was supported by grants from National Science Foundation (No. 39630140).

\section{REFERENCES}

[1] Frungieri MB, Gonzalez Galvar SI, Calandra RS. Influence of photoinhibition of GABA and glutamic acid levels, and on glutamate decarboxylase activity in the testis and epididymis of the golden hamster. Int J Andral 1996; 19(3):171-8.

[2] Ritta MN, Campos MB, Calandra RS. Coexistence of g-aminobutyric acid type A and type B receptors 
in testicular interstitial cells. J Neurochem 1991; 56(4):1236-40.

[3] Aanesen A, Fried G, Andersson E, Gottlieb C. Carrier-mediated g-aminobutyric acid uptake in human spermatozoa indicating the presence of a high-affinity g-aminobutyric acid transporter protein. Biol Reprod 1996; 54:841-6.

[4] Tam Dominic, Anthony CW, Lihe Guo. Cloning and sequencing of GABA transporter complementary. Cell Research 1994; 4(1):109-16.

[5] Guo Qiang Cai, Jian Fei, Yan Ping Xul et al. Nuclear proteins from liver and kidney bind a 37 bp sequence in the 5'upstream region of the mGAT1 gene. Neuroreport 1998; 9(18):4059-62.

[6] Markey CM, Jequier AM, Meyer GT, Martin GB. Testicular morphology and androgen profiles following testicular ischaemia in rams. J Repro Ferti 1994; 101:643-50.

[7] Krogsgaard-Larsen P, Falch E, Larssen OM, Schousboe A. GABA uptake inhibitors: relevance to antiepileptic drug research. Epilepsy Res 1987; 1:77-93.

Received Jan-24-2000. Revised Feb-15-2000. Accepted Feb-16-2000. 